\title{
Acute posterior vitreous detachment: the predictive value of vitreous pigment and symptomatology
}

Vaughan Tanner, Deacon Harle, James Tan, Barney Foote, Thomas H Williamson, Anthony H Chignell

\begin{abstract}
Aim-To establish whether the presence of a retinal break can be predicted either by the presence of a positive Shafer's sign (pigment granules in the anterior vitreous) or symptomatology in patients presenting with an acute posterior vitreous detachment (PVD).

Methods-200 eyes of 200 phakic patients with a symptomatic PVD of less than 1 month's duration underwent documentation of symptomatology and examination of the anterior vitreous for the presence of pigment granules. Indentation ophthalmoscopy was then carried out by an experienced vitreoretinal surgeon with no knowledge of the symptomatology or anterior vitreous gel examination findings. A second prospective group of 115 consecutive patients were assessed in a similar manner before primary rhegmatogenous retinal detachment repair.
\end{abstract}

Results-In 200 eyes presenting with an acute PVD, 25 were found to have an associated retinal break, 23 of which were also Shafer positive. In 115 eyes presenting for retinal detachment repair, 111 had an associated PVD and were found to be Shafer positive. Symptomatology was not predictive of an associated retinal break in the PVD group or in those presenting with a retinal detachment.

Conclusion-The increased use of Shafer's sign is recommended as a valuable aid in determining which patients require urgent referral for an expert retinal examination. It is not possible to predict those patients with a retinal break secondary to PVD on the basis of symptomatology alone.

(Br f Ophthalmol 2000;84:1264-1268)

It has been suggested that all patients older than 45 years of age presenting with symptoms of an acute posterior vitreous detachment (PVD) should undergo thorough retinal examination as a matter of urgency. ${ }^{1}$ Identification of any associated acute retinal breaks would allow application of photocoagulation or cryotherapy retinopexy to reduce the incidence of subsequent retinal detachment, which in untreated breaks occurs in approximately one third of cases. ${ }^{23}$

The incidence of PVD increases with age and myopia, ${ }^{4}$ and the urgent assessment of all patients with symptoms suggestive of PVD would constitute a significant increase in demand for ophthalmic services at short notice. It would be of benefit if those patients at particularly high risk of retinal break following PVD could be identified to allow more efficient use of ophthalmic resources. Previously identified high risk factors for the development of retinal breaks secondary to acute PVD include vitreous haemorrhage and the presence of unspecified vitreous cells. ${ }^{56}$ It has also been suggested that patients with symptoms of light flashes or multiple floaters may have a higher risk of an associated retinal break. ${ }^{67}$ However, others have reported a high incidence of retinal breaks in patients with only one or two isolated floaters as their presenting symptom. ${ }^{1}$

Previous studies have suggested that the presence of pigment cells in the anterior vitreous gel (Shafer's sign), ${ }^{8}$ following acute PVD, is strongly indicative of an associated retinal break. $^{9-12}$ The purpose of our prospective study was to further explore the correlation between a positive Shafer's sign and the presence of a retinal break in acute PVD. In order to further evaluate the correlation of Shafer's sign with retinal breaks, we have also prospectively evaluated a series of patients presenting with rhegmatogenous retinal detachment.

The ability to identify a subset of patients with underlying retinal breaks in those presenting with acute PVD would allow appropriate targeting of expert retinal examination on an urgent basis. In the UK most patients presenting with acute PVD symptoms are initially assessed by an optometrist in community practice or relatively inexperienced ophthalmologists in busy hospital emergency departments. These healthcare professionals may not feel confident in the practice of indirect indentation ophthalmoscopy and the exclusion of a retinal break, particularly if such breaks are small and in anterior retina. However, skills in examining the anterior vitreous with a slit lamp biomicroscope are more easily acquired. The use of Shafer's sign may be of great benefit in these circumstances to allow identification of those patients who should be referred on an emergency basis for more expert retinal examination and appropriate treatment.

\section{Methods}

POSTERIOR VITREOUS DETACHMENT SERIES

Case selection

Two hundred consecutive phakic patients were prospectively recruited over an 18 month period (April 1997 to October 1998) and 
assessed in a dedicated clinic at a London teaching hospital. Unselected referrals were made from the emergency department of our hospital, from local primary care physicians or from optometrists in the local community. Only patients over the age of 40 years and presenting with symptoms of an acute PVD within the previous month were recruited. Any patients with a history of previous retinal tear, other ocular disease, previous ocular surgery or symptoms longer than 1 month were excluded.

A validation of patient recruitment using a randomly selected subsample of patients attending the emergency department was undertaken to assess the completeness of the patient recruitment. Recruitment from community sources was encouraged through monthly written reminders.

\section{History and examination}

An experienced optometrist (DH) documented a detailed history of patient symptoms together with patient age and sex. Following formal refraction and Snellen visual acuity testing, the same optometrist then performed slit lamp biomicroscopy of the anterior segment and anterior vitreous of both eyes but did not examine the fundus. The optometrist recorded the presence or absence of anterior vitreous pigment with no knowledge of whether a retinal break was present. The presence of any other vitreous cells or haemorrhage was also recorded.

A standardised technique was used to perform dynamic examination of the anterior vitreous gel to ensure that any pigment in the gel was visualised. Following pupil dilatation, the anterior vitreous was first examined with the patient looking straight ahead using an oblique and narrow slit beam at high magnification. If vitreous pigment was not seen the patient was asked to saccade vertically and horizontally. This ocular movement set the vitreous gel in motion ensuring that any pigment cells in the peripheral gel, particularly those cells lying inferiorly, were visualised as the gel passed through the visual axis.

Each patient then underwent 360 degree indentation, indirect ophthalmoscopy by a vitreoretinal surgeon (VT, THW, or AHC), who was unaware of patient symptomatology or vitreous gel examination findings. The vitreoretinal surgeon then determined if a PVD was present using a hand held examination lens and slit lamp biomicroscopy of the posterior hyaloid face. A PVD was documented as being definitely present if a complete or partial ring of glial tissue (a Weiss ring) could be seen on the posterior hyaloid face. In our PVD series we included 27 patients who presented with classic history of acute PVD and at least partial detachment of the hyaloid face but no definite Weiss ring on examination. Although these patients can not be definitely confirmed to have had a complete separation of the posterior hyaloid face they are included as they would form a significant proportion of those patients presenting with PVD symptoms in clinical practice $(13.5 \%$ in this study).
If a retinal break was found retinopexy was then carried out using our usual techniques of argon laser or cryotherapy retinopexy. If a retinal detachment was found appropriate treatment was performed and the case was included in the retinal detachment series.

\section{RETINAL DETACHMENT SERIES}

Case selection

A second prospective group of 115 consecutive patients was recruited over the same period from patients presenting to our vitreoretinal unit for primary rhegmatogenous retinal detachment repair. Any patients with a history of retinopexy to a previous retinal tear, previous retinal detachment repair, retinal detachment due to trauma or retinal detachment associated with proliferative vitreoretinopathy were excluded. Any patient who had previously undergone any form of intraocular surgery including cataract surgery was also excluded.

\section{History and examination}

A history of symptoms before the development of retinal detachment was taken. Any symptoms reported as occurring more than 1 year before presentation were assumed not to be directly related to the current retinal detachment and excluded from subsequent analysis. All patients also underwent dynamic examination of the anterior vitreous gel, using the techniques described above, with emphasis on the detection of any pigment granules in the vitreous before surgery.

STATISTICAL ANALYSIS AND VALIDATION

All data were entered into a computer database and analysed using sPSs (Statistical Package for Social Sciences, 1995). Continuous variables were analysed using Student's $t$ test and the $\chi^{2}$ test was used for categorical variables.

Validation using a 1 month subsample of patients attending the emergency department identified that $8 / 12(67 \%)$ patients suitable for inclusion were referred to the study. Reasons for non-referral were non-intentional and identified as administration error or workload pressure. A comparison of the demographic and clinical features revealed no substantive differences between the two groups. Although there were no validation checks on the recruitment of patients through the community, the age and sex distribution was not significantly different from that of the emergency department group.

\section{Results}

POSTERIOR VITREOUS DETACHMENT SERIES Baseline data and symptomatology

Of 200 patients enrolled in the PVD series, there were 81 males and 119 females with a mean age of 61.6 years (range $41-84$ years). There was no significant difference $(\mathrm{p}=0.39)$ in mean male age (60.9 years) compared with mean female age (62.2 years). Eighty nine patients were referred from the emergency department of our hospital, 78 from local community optometrists, and 33 from local primary care physicians. The posterior vitreous detachment was symptomatic in the right eye 
Table 1 Presenting symptoms in patients from the PVD series

\begin{tabular}{lllll}
\hline & Floaters only & Flashes only & $\begin{array}{l}\text { Floaters } \\
\text { and flashes }\end{array}$ & $\begin{array}{c}\text { Blurring } \\
\text { of vision }\end{array}$ \\
\hline PVD series, no retinal break $(\mathrm{n}=175)$ & $68(38.8 \%)$ & $25(14.3 \%)$ & $80(45.7 \%)$ & $2(1.4 \%)$ \\
PVD series, retinal break present $(\mathrm{n}=25)$ & $15(60 \%)$ & $1(4 \%)$ & $9(36 \%)$ & 0 \\
\hline PVD = posterior vitreous detachment. & & & & \\
Percentages given to one decimal place. & & &
\end{tabular}

Table 2 Vitreous examination findings in PVD and RD series

\begin{tabular}{llcc}
\hline & $\begin{array}{l}\text { PVD and } \\
\text { retinal break } \\
(n=25)\end{array}$ & $\begin{array}{l}\text { PVD no } \\
\text { retinal break } \\
(n=175)\end{array}$ & $\begin{array}{l}\text { RD } \\
(n=113)\end{array}$ \\
\hline Shafer positive & 23 & 0 & 111 \\
Shafer negative & 2 & 175 & 2 \\
Haemorrhage present & 0 & 10 & 0 \\
White cells present & 0 & 8 & 0 \\
\hline
\end{tabular}

PVD $=$ posterior vitreous detachment $\mathrm{RD}=$ retinal detachment.

in 112 patients and the left in 88 patients. One patient presented with bilateral symptoms and the right eye was randomly assigned to be the study eye. Mean refraction of symptomatic eyes was -1.2 dioptres spherical equivalent (range -21.5 to +5 dioptres), a total of 78 eyes (39\%) eyes had a myopic spherical equivalent.

Presenting symptoms and vitreous findings for the PVD series are shown in Table 1. The presenting symptom in the PVD series was floaters alone in 83 eyes, light flashes alone in 26 eyes, floaters and light flashes together in 89 eyes, and vague blurring of vision in two eyes. Symptom duration was grouped as follows: 2 days or less 14 eyes, 1 week or less 56 eyes, 1 week to 1 month 130 eyes.

\section{Shafer's sign}

A total of $177(88.5 \%)$ eyes were classified as Shafer negative and $23(11.5 \%)$ eyes as Shafer positive as shown in Table 2 . In those patients who were Shafer positive the average age was 57.5 years, there were 12 males and 11 females with an average refraction of -1.7 dioptres spherical equivalent. The presenting complaint in Shafer positive eyes was floaters alone in 15 eyes, light flashes alone in one eye, and light flashes and floaters together in seven eyes. Out of the total 200 eyes, retinal tear was present in 17 eyes, retinal hole in eight eyes and lattice degeneration in seven eyes. All 23 patients who were Shafer positive had an associated retinal tear or hole and symptom duration was grouped as follows: 2 days or less six eyes, 1 week or less five eyes, 1 week to 1 month 12 eyes.

Two patients were found to be Shafer negative despite a retinal tear or hole and are described in detail below.

Case 1

A 64 year old man presented with a 3 week history of floaters and light flashes in his right eye.
Spherical equivalent in this eye was -2.00 , a definite Weiss ring was present. No pigment was seen in the anterior vitreous gel, fundal examination revealed a small, round, chronic hole in the temporal periphery with an associated free floating operculum and extensive subretinal pigmentation around the hole margins. No retinopexy was carried out as the break was felt to be long standing.

Case 2

A 69 year old woman presented with a 4 day history of floaters and light flashes in her left eye. Spherical equivalent was +4.00 dioptres and a definite Weiss ring was present. No pigment was seen in the anterior vitreous gel, fundal examination revealed a small superotemporal fresh horseshoe retinal tear which was treated with laser retinopexy.

Of the 200 eyes, vitreous haemorrhage was present in 10 eyes and a Weiss ring was seen in 173 eyes. Eight eyes were noted to have several white cells in the anterior vitreous gel, all of these eyes were Shafer negative and none had an associated retinal break.

\section{RETINAL DETACHMENT SERIES}

Baseline data and symptomatology

Of 115 patients enrolled in the retinal detachment (RD) series, there were 65 males and 50 females with a mean age of 57 years (range $13-88$ years). There was no significant difference $(p=0.64)$ in mean male age (56 years) compared with mean female age (58.2 years). Refractive error was myopic in $56(48.7 \%)$.

A total of 68 patients presented within 1 month of onset of symptoms, 31 of these patients presented within 1 week of onset of symptoms. The mean duration from onset of any symptoms to presentation was 63 days. Presenting symptoms for the RD series are shown in Table 3. The majority of patients in the $\mathrm{RD}$ series, 105 eyes (91.3\%), presented with visual or field loss, either in conjunction with flashes or floaters in 83 eyes $(72.2 \%)$ or alone in $22(19.1 \%)$ eyes. Of the 83 eyes presenting with additional flashes or floaters, a total of 59 patients gave a definite history of sudden onset of flashes or floaters before the development of a visual field defect or visual loss. Other presentations included floaters alone in four $(3.4 \%)$ eyes, flashes alone in three $(2.6 \%)$, flashes and floaters only in one $(0.8 \%)$ eye, and asymptomatic in two $(1.7 \%)$ eyes.

\section{Shafer's sign}

A total of 111 eyes were classified as Shafer positive as shown in Table 2 . In one eye the anterior vitreous could not be seen clearly because of dense cataract and in one eye the Shafer sign was not documented. Therefore, two eyes were Shafer negative in the presence

Table 3 Presenting symptoms in patients from the retinal detachment series

\begin{tabular}{|c|c|c|c|c|c|c|c|}
\hline & $\begin{array}{l}\text { Field or visual loss } \\
\text { preceded by flashes } \\
\text { or floaters }\end{array}$ & $\begin{array}{l}\text { Field or visual loss } \\
\text { with simultaneous } \\
\text { flashes or floaters }\end{array}$ & $\begin{array}{l}\text { Field or visual } \\
\text { loss alone }\end{array}$ & Floaters only & Flashes only & $\begin{array}{l}\text { Floaters } \\
\text { and flashes }\end{array}$ & Asymptomatic \\
\hline No of eyes (total $=115)$ & $59(51.3 \%)$ & $24(20.9 \%)$ & $22(19.1 \%)$ & $4(3.4 \%)$ & $3(2.6 \%)$ & $1(0.8 \%)$ & $2(1.7 \%)$ \\
\hline
\end{tabular}

Percentages given to one decimal place. 
of a retinal detachment. Neither of these patients had premonitory symptoms, their retinal detachments having been discovered following a routine eye examination. Neither patient had developed a posterior vitreous detachment and the retinal detachment in each case was inferior, long standing, and secondary to round retinal holes in the absence of lattice degeneration.

\section{Discussion}

The incidence of retinal break formation following acute posterior vitreous detachment is variously reported as occurring in between $8 \%$ to $15 \%$ of patients in studies similar to ours. ${ }^{5}{ }^{10}$ The incidence of retinal break formation in our PVD series of $12.5 \%$ is therefore consistent with that previously reported.

Of 200 patients who presented with an acute PVD, a significantly greater proportion were female $(59.5 \%$ \% $40.5 \%, \mathrm{p}=0.003)$. Byer ${ }^{1}$ has suggested that the apparent higher frequency of PVD in females is the result of the higher incidence of females in the general population at the age of PVD. He supported this theory with a comparative control group drawn from new patients presenting to his practice, and found no statistically significant difference in sex between this control group and those presenting with PVD. However, of 115 patients presenting for RD repair, 65 were male and 50 female $(\mathrm{p}=0.007)$, and of the 25 patients in our PVD series who were found to have retinal breaks there were 13 males $(16 \%)$ and 12 females $(10 \%)$. This significant association between male sex and PVD being associated with a retinal break or of presenting with a rhegmatogenous $\mathrm{RD}\left(\chi^{2}=13.54 \mathrm{p}<0.001\right)$ is similar to the reports of several other authors including Byer, but has no obvious explanation. ${ }^{561013}$

Despite careful history taking it was not possible on the basis of symptoms alone to determine which patients had a retinal break consequent to a PVD, a finding confirmed by previous authors. ${ }^{1}{ }^{10}$ Some authors have suggested that vitreous floaters alone carry a much less significant risk of retinal tear than when present in conjunction with flashes. ${ }^{714}$ It is interesting that in our PVD series 83 eyes presented with floaters only, of which 15 eyes had associated retinal breaks, and 89 eyes presented with a combination of flashes and floaters, of which nine eyes had associated retinal breaks. Therefore, symptoms of floaters alone was associated with a retinal break in $18.1 \%$ of cases and symptoms of flashes and floaters together was associated with a retinal break in $10.1 \%$ of cases. The combination of flashes and floaters together did not have a significantly higher chance $\left(\chi^{2}=1.65, \mathrm{p}=0.19\right)$ of being associated with a retinal tear than floaters alone. In our series it was not possible to determine on the basis of symptoms alone which patients with an acute PVD were more likely to have an associated retinal break and need urgent expert retinal examination.

In our series of 115 patients presenting with retinal detachment 59 patients reported definite onset of symptoms of floaters or flashes before the onset of field or visual loss, four patients presented with floaters alone, three with flashes alone, and one with floaters and flashes. It is interesting to note that 22 patients (19.1\%) presented with field or visual loss and no other symptoms. While emphasising the importance of expert retinal examination in patients presenting with symptoms of PVD these observations do not help in determining which symptoms are more likely to be associated with a retinal break or detachment.

However, we have found the presence of pigment in the vitreous gel to be a reliable indicator of the presence of a retinal break in association with an acute PVD. occurring in 23/25 $(92 \%)$ patients. We did not detect pigment in the vitreous gel of any patient who did not have a retinal break. Of the two patients in the PVD series with retinal breaks who did not have vitreous pigment, one was found to have a round hole present in an area of lattice type retinal degeneration. We suspect that the hole had been present for a significant time before the development of PVD since there was extensive pigmentation around the hole margins. If this patient is excluded the correlation between vitreous pigment and retinal break rises to $23 / 24$ giving a sensitivity of $95.8 \%$ and a specificity of $100 \%$.

The positive association between vitreous pigment and retinal break was confirmed by our study of those patients presenting with retinal detachment. All patients with symptomatic retinal detachment in whom Shafer's sign was documented were found to have vitreous pigment. Interestingly, vitreous pigment was absent in two patients in the retinal detachment group, both of whom had asymptomatic retinal detachments secondary to round holes not associated with lattice degeneration in the absence of a PVD. The pathogenesis of break formation in these patients is different to that occurring in acute retinal breaks secondary to vitreoretinal traction following PVD. Either this process does not result in the release of pigment or only a small amount of pigment is released. It is also possible that the absence of a posterior vitreous detachment inhibits dispersion of pigment through the gel. It is important to emphasise that dynamic vitreous gel examination should be performed as the amount of vitreous pigment present is often small and may settle inferiorly making it difficult to see unless the vitreous gel is set in motion. ${ }^{15}{ }^{16}$

Pigment appears in the anterior vitreous gel almost immediately after break formation, and was seen in retinal tears of only a few hours' duration. It is important to emphasise that the strong positive correlation found in our study between vitreous pigment and retinal tear may apply only to those patients with acute PVD of recent onset. However, this group of patients comprises those at greatest risk of retinal detachment secondary to a retinal break. Our series is also not a community based study and it is therefore difficult to completely exclude a possible referral bias in patients presenting to our tertiary referral practice, despite our attempts to encourage referral of all patients with acute PVD on an unselected basis. 
Brod et $a l^{10}$ have performed a similar study to ours looking at 106 patients with acute PVD and also found a high correlation between vitreous pigment and retinal break with 15 eyes having pigment cells in the vitreous gel out of 16 eyes with a retinal tear. Other retrospective studies of patients presenting with retinal detachments have found a weaker correlation $\left(83 \%,{ }^{9} 93 \%{ }^{17}\right)$.

Of 200 patients in the PVD series we identified eight patients with an unusual number of white cells within their vitreous but no other evidence of intraocular inflammation and no retinal break. It is therefore important to note the colour as well as presence of vitreous cells when trying to assess the risk of an underlying retinal break. It may be occasionally difficult to differentiate red blood cells from pigmented cells in the vitreous, although the pigment cells usually appear larger, darker and more irregular in shape. We were able to differentiate pigment cells from red blood cells in one of our patients who presented with a retinal tear and associated mild vitreous haemorrhage, but this may be more difficult in cases where the vitreous haemorrhage is particularly dense.

We therefore believe the use of Shafer's sign offers an extremely valuable aid in the examination of patients with acute PVD. If vitreous pigment is seen in the absence of previous ocular surgery it should be assumed a retinal break is present and the patient should be referred immediately for expert retinal examination and treatment. We recommend that those patients with acute PVD and no vitreous pigment be referred for expert opinion on a less urgent basis. Further examination is probably still necessary owing to the occasional difficulty in observing very small amounts of vitreous pigment in some patients with small retinal tears, the occasional patient who has an acute retinal tear in the absence of vitreous pigment, and the possible, rare, occurrence of delayed retinal breaks.

1 Byer NE. Natural history of posterior vitreous detachment with early management as the premier line of defence against retinal detachment [see?. Ophthalmology 1994;101:1503-13.

2 Robertson MD, Norton EWD. Long term follow up of treated retinal breaks. Am Ophthalmol 1973;75:395-404.

3 Davies MD. Natural history of retinal breaks without detachment. Arch Ophthalmol 1974;92:183-94.

4 Akiba J. Prevalence of posterior vitreous detachment in high myopia. Ophthalmology 1993;100:1384-8.

5 Novak MA, Welch RB. Complications of acute symptomatic posterior vitreous detachment. Am Ophthalmol 1984;97: posterio

6 Boldrey EE. Risk of retinal tears in patients with vitreous floaters. Am Ophthalmol 1983;96:783-7.

7 Hikichi T, Trempe CL. Relationship between floaters, light flashes, or both, and complications of posterior vitreous detachment. Am Ophthalmol 1994;117:593-8.

8 Shafer DM, Stratford DP. Binocular indirect ophthalmoscopy. In: Schepens CL, Regan CDJ, eds. Controversial aspects of the management of retinal detachment. London: J \& A Churchill, 1965:51.

9 Hamilton AM, Taylor W. Significance of pigment granules in the vitreous. Br f Ophthalmol 1972;56:700-2.

10 Brod RD, Lightman DA, Packer AJ, et al. Correlation between vitreous pigment granules and retinal breaks in eyes with acute posterior vitreous detachment. Ophthalmology 1991;98:1366-9.

11 Lightman DA, Brod RD. Relationship between floaters, light flashes, or both, and complications of posterior vitreous detachment [letter; comment]. Am Ophthalmol 1994; ous detach

12 Sharma S, Walker R, Brown GC, et al. The importance of qualitative vitreous examination in patients with acute posterior vitreous detachment. Arch Ophthalmol 1999;117: 343-6.

3 Jaffe NS. Complications of acute posterior vitreous detachment. Ophthalmology 1968;79:568-71.

14 Diamond JP. When are simple flashes and floaters ocular emergencies? Eye 1996;6:102-4.

15 Rosen E. The ascension phenomenon of the anterior vitreous. Am Ophthalmol 1962;53:55-65.

16 Takahashi M, Trempe CL Schepens. Biomicroscopic evaluation and photography of posterior vitreous detachment. ation and photography of poste
Arch Ophthalmol 1980;98:665-8.

17 Bartkowska-Orlowska M, Szczypinski J. Pigment granules in the vitreous diacritical body in uncomplicated retinal detachment. Klin Oczna 1974;14:1043-7. 